\title{
Inhibitory effect of midazolam on MMP-9, MMP-1 and MMP-13 expression in PMA-stimulated human chondrocytes via recovery of NF- $\kappa \mathrm{B}$ signaling
}

\author{
Jen-Jui Wang', Steven Kuan-Hua Huan², Kuo-Hsien Hsieh³, Hsiu-Chu Chou, George Hsiao5, \\ Thanasekaran Jayakumar ${ }^{5}$, Joen-Rong Sheu ${ }^{5}$
}

1Department of Anesthesiology, West Garden Hospital, Taipei, Taiwan

2Division of Urology, Department of Surgery, Chi-Mei Medical Center, Tainan, Taiwan

3Tung's Taichung Metro Harbor Hospital, Taichung, Taiwan

4Department of Anatomy, and Graduate Institute of Medical Sciences, Taipei Medical University, Taiwan

${ }^{5}$ Department of Pharmacology and Graduate Institute of Medical Sciences, Taipei Medical University, Taiwan

Submitted: 22 August 2011

Accepted: 5 December 2011

Arch Med Sci 2013; 9, 2: 332-339

DOI: $10.5114 /$ aoms.2012.30949

Copyright (C 2013 Termedia \& Banach

\section{Abstract}

Introduction: Midazolam, a benzodiazepine, has a hypnotic effect and is widely used as an intravenous sedative. Past studies have clearly established that midazolam has beneficial effects in attenuating ischemia-reperfusion injury more than other currently used sedative drugs. However, the role of midazolam on chondroprotection via inhibition of matrix metalloproteinases (MMPs) is warrant investigation. The aim of this study was to examine the mechanisms of action of midazolam on MMP expression via nuclear factor $\kappa B(N F-\kappa B)$ signaling in activated chondrosarcoma cells maintained in vitro.

Material and methods: Chondrocytes, SW1353 cells, were stimulated with phorbol 12-myristate 13-acetate (PMA) in the absence or presence of various concentrations of midazolam $(5-20 \mu \mathrm{M})$. Release of MMP-9 into the culture media was determined by gelatin zymography. The expressions of MMP-1, MMP-9 and MMP-13, phosphorylation of extracellular signal-regulated kinase (ERK), p38 mitogen-activated protein kinases and degradation of $\mid \kappa B-\alpha$ were determined by western blotting assay.

Results: Midazolam significantly down-regulated PMA-induced MMP-9 protein expression at concentrations of 5,10 and $20 \mu \mathrm{M}$, the values were $1.95 \pm 0.09$ ( $p<0.01), 1.71 \pm 0.12(p<0.01)$ and $1.35 \pm 0.20$ ( $p<0.001)$, respectively. At concentrations of 5,10 and $20 \mu \mathrm{M}$, it was significantly inhibited the PMA-induced expressions of MMP-1 $(2.27 \pm 0.10,1.98 \pm 0.11$ and $1.56 \pm 0.15 ; p<0.001)$ and MMP-13 $(0.89 \pm 0.04,0.81 \pm 0.07$, and $0.74 \pm 0.09 ; p<0.001)$, respectively. Midazolam at concentrations of 10 and $20 \mu \mathrm{M}$ for 15 min significantly reversed the rate of degradation (0.895 $\pm 0.051 ; p<0.05$ and $0.926 \pm 0.060 ; p<0.01$, respectively) of $I_{\kappa B}-\alpha$ in PMA-chondrocyte cells. In addition, this sedative drug inhibited PMAinduced levels of phos-ERK $(1.243 \pm 0.12,1.108 \pm 0.16$ and $0.903 \pm 0.19$, respectively) and phos-p38 (1.146 $\pm 0.10,1.063 \pm 0.13$ and $0.946 \pm 0.18$, at concentrations of $(5,10$ and $20 \mu \mathrm{M})$, respectively.

Conclusions: These results are important for understanding the mechanism of midazolam in inhibiting PMA-induced MMP expression through the signaling pathways of either NF-KB or ERK/p38 MAPKs down-regulation.

Key words: human chondrosarcoma cells, midazolam, phorbol 12-myristate 13-acetate, matrix metalloproteinases, mitogen-activated protein kinases, IкB- $\alpha$.

\author{
Corresponding author: \\ Dr. Joen-Rong Sheu \\ Graduate Institute \\ of Medical Sciences \\ Taipei Medical University \\ $250 \mathrm{Wu}$-Hsing St \\ Taipei 11031, Taiwan \\ Phone: +886-2-27361661 \\ ext. 3199 \\ Fax: +886-2-27390450 \\ E-mail: sheuj@@tmu.edu.tw \\ Dr. Thanasekaran Jayakumar \\ Department of Pharmacology \\ Taipei Medical University \\ 250 Wu-Hsing St \\ Taipei 11031, Taiwan \\ Phone: +886-2-27361661 \\ ext. 3205 \\ E-mail: tjaya_2002@yahoo.co.in
}




\section{Introduction}

Osteoarthritis $(\mathrm{OA})$ is a joint disease that involves degeneration of the articular cartilage and the formation of bone at the joint surface and margins. Although OA differs from rheumatoid arthritis (RA) in that it is not a systemic disease, synovitis in OA does occur, although more frequently in the advanced stages. Osteoarthritis cartilage is a rich source of inflammatory mediators, including cytokines, nitric oxide, and prostaglandins [1]. A role for proinflammatory cytokines in the pathogenesis of OA was proposed based on the data from studies using human OA cartilage and various animal models [2]. Cytokine production associated with degenerative changes in OA cartilage has been reported in a study [3], in which interlekin-1 $\alpha$ (IL-1 $\beta$ ) and tumour necrosis factor- $\alpha$ (TNF- $\alpha$ ) were shown to co-localize with matrix metalloproteinases in the superficial zones of articular cartilage. Phorbol 12-myristate 13-acetate (PMA) was reported to be involved in expression of matrix metalloproteinases (MMPs) in activated human chondrocytes [4].

The metalloproteinases are a large group of enzymes that play a crucial role in tissue remodeling as well as in the destruction of cartilage and bone in an arthritic joint due to their ability to degrade a wide variety of extracellular matrix (ECM) components [5]. These proteolytic enzymes attack and degrade components of the extracellular matrix. The MMPs involved in endochondral ossification are: the collagenases, MMP-1 and MMP-13; the gelatinases, MMP-2 and MMP-9 (gelatinase A and $B$, respectively); the stromelysins, MMP-3 and MMP-10; and the membrane-type MMP-14 [6]. Inflammatory cytokines, such as IL-1 $\beta$ and TNF- $\alpha$, have been reported to stimulate inducible expression of MMPs (1, 3, 9 and 13) in cartilage [7]. MMP-1 (collagenase-1) is expressed ubiquitously and is found in various cells, including chondrocytes [8]. MMP-13 (collagenase-3) has long been regarded as the major source of collagen degrading activity, since it has preferential capacity to degrade type II collagen [9]. Our recent study has demonstrated that PMA, as a protein kinase C (PKC) activator, stimulates human chondrocytes via expression of matrix metalloproteinases (MMPs) [10]. One study has well described that the mode of PKC mediated signaling involves transmission of signals from PKC to mitogen-activated protein kinases (MAPKs).

It is also well documented that the MAP kinase family members including extracellular signalregulated kinase (ERK), p38 MAP kinases and c-Jun $\mathrm{N}$-terminal kinase (JNK) are known to be activated in human chondrocytes [11]. I $1 \kappa \mathrm{B}-\alpha$ is reported to play a significant role in arthritis patients due to its inhibition of MMP-1 and MMP-13 production [12]. A variety of inhibitors of the MMPs are currently being used. However, adverse drug effects, particularly gastrointestinal ulceration, are commonly associated with these agents. Therefore, currently there is much interest in developing more effective and physiologic approaches such as therapeutic use of biological agents that block the activity of MMPs.

Of the benzodiazepines (BDZ), midazolam is the most commonly administered intravenous sedative in clinical practice and in intensive care medicine. The analgesic property of midazolam has been reported to be mediated via the $\gamma$-aminobutyric acid (GABA) A receptor in the spinal cord [13]. Furthermore, both human and animal models have shown ubiquitous distribution of peripheral BDZ receptors (PBRs) in various organs and in joints [14]. A study has also proved that PBR is highly localized in chondrocytes [15]. Midazolam has gained widespread use in cardiac surgery; its cardioprotective effects have been increasingly recognized. Experimental and clinical studies have demonstrated that midazolam attenuated myocardial ischemia-reperfusion injury [16]. Although midazolam has more beneficial effects than other currently used sedative drugs [17], its effects on chondrocytes have not been investigated at the cellular or molecular levels.

Hence, the aim of this study was to use an in vitro model of human chondrocytes to study the effects of midazolam on MMP expression and test the hypothesis that midazolam antagonizes the effect of PMA by suppressing MAPK expression.

\section{Material and methods}

\section{Material}

Midazolam, sodium dodecyl sulfate (SDS), phenylmethylsulfonyl fluoride (PMSF), leupeptin, aprotinin, sodium fluoride, sodium orthovanadate, sodium pyrophosphate, diethyl pyrocarbonate (DEPC), phorbol 12-myristate 13-acetate (PMA), and bovine serum albumin (BSA) were all purchased from Sigma-Aldrich (St. Louis, MO, USA). Anti-mouse and antirabbit immunoglobulin G (lgG)-conjugated horseradish peroxidase (HRP) was purchased from Amersham Biosciences (Sunnyvale, CA) and/or Jackson-Immuno Research (West Grove, PA). A mouse monoclonal antibody (mAb) specific for human native 92-kDa MMP-9 was purchased from LabVision/NeoMarkers (Fremont, CA). A rabbit polyclonal antibody (pAb) specific for $1 \kappa B-\alpha$ was purchased from Santa Cruz Biotechnology (Santa Cruz, CA). The anti-p38 MAPK and anti-phospho-c-Jun N-terminal kinase (JNK) (Thr183/Tyr185) mAbs, and the anti-phospho p44/p42 extracellular signal-regulated kinase (ERK) (Thr202/ Tyr204) polyclonal antibody were from Cell Signaling (Beverly, MA, USA); and the Hybond-P PVDF membrane, ECL Western blotting detection reagent and analysis system were from Amersham (Buckinghamshire, UK). All other chemicals used in this study were of reagent grade. Midazolam was dis- 
solved in $0.5 \%$ dimethyl sulfoxide (DMSO) and stored at $4^{\circ} \mathrm{C}$ until use.

\section{Cell cultivation}

Human chondrosarcoma cells, SW1353, were obtained from American Type Culture Collection (Manassas, VA). Cells were cultured in Ham's F-12 and Dulbecco's Modified Eagle Medium (DMEM) $(1: 1)$ supplemented with L-glutamine $(3.65 \mathrm{mM})$, penicillin $(90 \mathrm{U} / \mathrm{ml})$, streptomycin $(90 \mu \mathrm{g} / \mathrm{ml})$, HEPES (18 mM), $\mathrm{NaHCO}_{3}(23.57 \mathrm{mM})$, and $10 \%$ heat-inactivated fetal bovine serum (FBS) at $37^{\circ} \mathrm{C}$ in humidified air with $5 \% \mathrm{CO}_{2}$.

\section{Stimulation experiments}

For stimulation of PMA, chondrosarcoma SW1353 cells were seeded at $2.5 \times 10^{6}$ per well of Costar 6 -well tissue culture plates in complete media until a confluence of $85 \%$ was reached (usually for $24 \mathrm{~h}$ ). After $24 \mathrm{~h}$, cells were changed to serum free media. Cells were treated with midazolam $(5-20 \mu \mathrm{M})$ for $15 \mathrm{~min}$ after $24 \mathrm{~h}$ of changing serum free media and then treated with PMA $(10 \mathrm{ng} / \mathrm{ml})$ for an another $24 \mathrm{~h}$. At the end of the incubation period, conditioned medium and cell supernatants were collected and stored at $-80^{\circ} \mathrm{C}$ for gelatin zymography and Western blotting assay, respectively.

\section{Cell viability assay}

The cytotoxic effects of midazolam against the SW1353 cell line were determined by the MTT method as described previously [18]. Briefly, cells $\left(2 \times 10^{6}\right.$ cells $\left./ \mathrm{ml}\right)$ were incubated in 12-well plates with different concentrations $(5-30 \mu \mathrm{M})$ of midazolam for $24 \mathrm{~h}$ at $37^{\circ} \mathrm{C}$. At the $22^{\text {nd }} \mathrm{h}$, the MTT solution was added to each well at a final concentration of $0.5 \mathrm{mg} / \mathrm{ml}$. After 2 -h incubation at $37^{\circ} \mathrm{C}$, the supernatant was discarded and replaced with DMSO to dissolve the formazan product, which was measured at $550 \mathrm{~nm}$ in a spectrophotometric plate reader. The following formula was used to calculate the \% cell viability: Percentage cell viability = (absorbance of the experiment samples/absorbance of the control) $\times 100 \%$.

\section{Gelatin zymography}

The expression of MMP-9 was detected by gelatin zymography as described by Chung et al. [19]. The conditioned medium was mixed with nonreducing buffer (500 mM Tris- $\mathrm{HCl}, 25 \%$ glycerol, 10\% SDS, and $0.32 \%$ bromophenol blue; $\mathrm{pH} 6.8$ ) and electrophoresed in $10 \%$ polyacrylamide gel containing gelatin $(1 \mathrm{mg} / \mathrm{ml})$. After electrophoresis, the gels were washed 2 times with $2.5 \%$ Triton X-100 to remove the SDS and then incubated with reacting buffer containing $50 \mathrm{mM}$ Tris-base, $200 \mathrm{mM} \mathrm{NaCl}$,

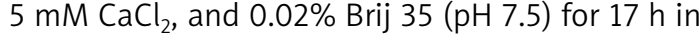
a closed container at $37^{\circ} \mathrm{C}$. At the end of the incubation, the gels were fixed with a fixing solution ( $7 \%$ acetic acid and $40 \%$ methanol, v/v) for $30 \mathrm{~min}$. Gels were stained with a solution of Colloidal Brilliant Blue G in $27 \%$ methanol for 30 min or longer. Finally, a destaining solution (10\% acetic acid in $25 \%$ methanol) was used to adjust the clear conditions. Clear zones (bands) against the blue background indicated the presence of degradative activity of MMP-9.

\section{SDS-polyacrylamide gel electrophoresis and Western blot analysis}

Western blot analyses were performed as previously described [18]. Lysates from each sample were mixed with $6 \times$ sample buffer $(0.35 \mathrm{M}$ Tris, $10 \% \mathrm{w} / \mathrm{v}$ SDS, $30 \% \mathrm{v} / \mathrm{v}$ glycerol, $0.6 \mathrm{M} \mathrm{DTT}$, and $0.012 \% \mathrm{w} / \mathrm{v}$ bromophenol blue, $\mathrm{pH}$ 6.8) and heated to $95^{\circ} \mathrm{C}$ for 5 min. Proteins were separated by electrophoresis and transferred onto nitrocellulose membranes (for MMP-9) and polyvinylidene difluoride (PVDF) membranes (for MMP-1/-13, p38, pERK1/2, c-JUN and $\mid \kappa B-\alpha)$. The membranes were blocked with $5 \%$ nonfat milk in TBS-0.1\% Tween 20 and sequentially incubated with primary antibodies and HRP-conjugated secondary antibodies, followed by enhanced chemiluminescence (ECL) detection (Amersham Biosciences). The BIO-PROFIL Bio-1D light analytical software (Vilber Lourmat, Marue La Vallee, France) was used for the quantitative densitometric analysis. Data of specific protein levels are presented as relative multiples in relation to the control.

\section{Statistical analysis}

The experimental results are expressed as the mean \pm SEM and are accompanied by the number of observations. For analysis of the results, a one-way analysis of variance (ANOVA) test was performed using the Sigma Stat v3.5 software. When group comparisons showed a significant difference, the StudentNewman-Keuls test was used. A value of $p$ less than 0.05 was considered statistically significant.

\section{Results}

\section{Effects of midazolam on MMP-9 activation and expression}

To investigate the effects of midazolam on MMP-9 expression and activation, the human chondrosarcoma cell line (SW1353) was exposed to various concentrations of midazolam $(5-20 \mu \mathrm{M})$ and then stimulated with PMA (10 ng/ml). A marked increase $(3.08 \pm 0.21 ; p<0.001)$ in the activation of MMP-9 was noticed after $24 \mathrm{~h}$ of PMA stimulation as compared with the control (0.5\%, DMSO). However, treatment of SW1353 cells with midazolam ( $5 \mu \mathrm{M}, 10 \mu \mathrm{M}$ 
and $20 \mu \mathrm{M}$ ) was found to down-regulate PMAmediated MMP-9 gelatinolysis approximated at $2.91 \pm 0.19,2.61 \pm 0.11$ and $2.23 \pm 0.20$, respectively (Figure $1 \mathrm{~A}$ ). Similarly, a significant induction of MMP-9 protein expression $(2.18 \pm 0.17)$ by PMA $(10 \mathrm{ng} / \mathrm{ml})$ was found to be down-regulated in midazolam $(5 \mu \mathrm{M}, 10 \mu \mathrm{M}$ and $20 \mu \mathrm{M})$ pretreated cells; the values were $1.95 \pm 0.09$ ( $p<0.01), 1.71 \pm 0.12$ ( $p<0.01)$ and $1.35 \pm 0.20$ ( $p<0.001)$, respectively (Figure $1 \mathrm{~B})$.

To further evaluate whether midazolam inhibits MMP-9 activation in SW1353 cells through cytotoxic effects, cells were pre-incubated with midazolam (5-30 $\mu \mathrm{M})$ for $24 \mathrm{~h}$. Based on the MTT assay, it was found that this sedative drug had little effect on cell viability of SW1353 cells, even at a higher concentration $(30 \mu \mathrm{M})$ at about $84.16 \pm 1.49 \%$ (data not shown).

\section{Up-regulation of MMP-1 and MMP-13 expression by PMA in chondrocytes is inhibited by midazolam}

To evaluate MMP-1 and 13 expression in response to PMA followed by midazolam treatment, chondrocyte cultures were serum starved for $24 \mathrm{~h}$ and then stimulated with PMA $(10 \mathrm{ng} / \mathrm{ml})$ in the presence or absence of midazolam (5-20 $\mu \mathrm{M})$. The results shown in Figure $2 \mathrm{~A}$ indicate that, at concentrations of $5 \mu \mathrm{M}, 10 \mu \mathrm{M}$ and $20 \mu \mathrm{M}$, midazolam could significantly inhibit the activation of MMP-1 $(2.27 \pm 0.10,1.98 \pm 0.11$ and $1.56 \pm 0.15 ; p<0.001)$, respectively) induced by PMA $(2.42 \pm 0.19)$. A similar significantly lower expression $(0.89 \pm 0.04,0.81$ \pm 0.07 , and $0.74 \pm 0.09 ; p<0.001)$ of MMP-13 was noted in midazolam $(5 \mu \mathrm{M}, 10 \mu \mathrm{M}$ and $20 \mu \mathrm{M}$, respectively) pretreated and PMA-induced (1.31 \pm 0.04 ) SW1353 cells (Figure $2 \mathrm{~B}$ ). These results indicate that midazolam down-regulates the stimulated expression of MMP-1 and MMP-13 in a concentration-dependent manner.

\section{Midazolam inhibits PMA-mediated degradation of $I_{\kappa} B-\alpha$}

To determine whether the inhibitory action of midazolam was due to an effect on degradation, the cytoplasmic levels of $I_{\kappa} B-\alpha$ protein were examined by Western blot analysis. As shown in Figure 3, treatment of cells with PMA (for $30 \mathrm{~min}$ ) could induce a rate of degradation of $\mathrm{I}_{\mathrm{K} B} \mathrm{~B}-\alpha$ of about 0.786 \pm 0.047 . Interestingly, SW1353 cells that had been treated with midazolam at concentrations of $10 \mu \mathrm{M}$ and $20 \mu \mathrm{M}$ for 15 min significantly reversed the rate of degradation ( $0.895 \pm 0.051 ; p<0.05$ and 0.926 $\pm 0.060 ; p<0.01$, respectively) of $1 \kappa \mathrm{B}-\alpha$ induced by PMA (Figure 3). However, midazolam treatment at a concentration of $5 \mu \mathrm{M}$ did not significantly recover the rate of PMA-mediated degradation of $I_{\kappa} B-\alpha$.
A
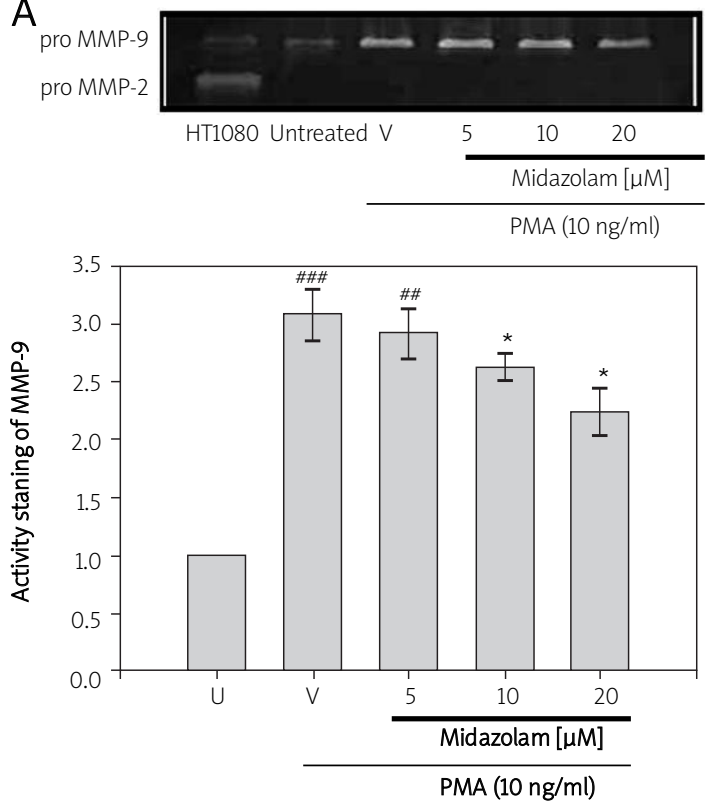

B
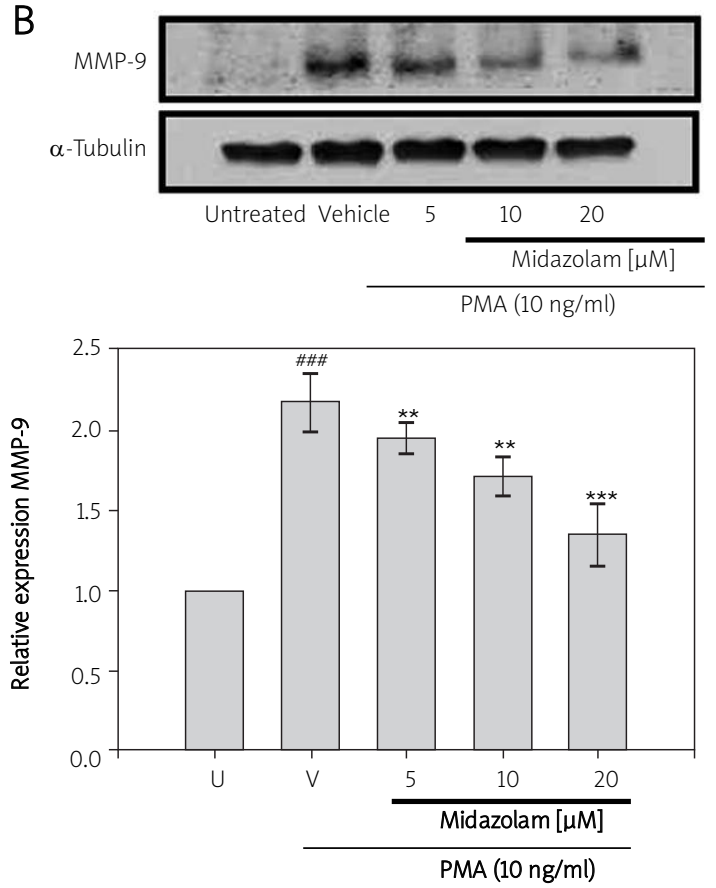

Figures 1. Effects of midazolam on phorbol-12myristate-13-acetate (PMA)-induced activation and expression of MMP-9 in chondrosarcoma SW1353 cell lines. Cell-free conditioned medium and supernatants were assayed for MMP-9 activation and expression by gelatin zymography $(\mathrm{A})$ and western blotting (B), respectively. Lane 1 is the culture medium of HT1080 cells in which the constitutive proMMP-2 and proMMP9 were expressed (A). SW1353 cells $\left(2 \times 10^{6}\right.$ cells $/ \mathrm{ml}$ in 6-well plates) were treated with various concentrations of midazolam $(5,10$, and $20 \mu \mathrm{M})$ or vehicle (DMSO) for $15 \mathrm{~min}$ before treatment with PMA $(10 \mathrm{ng} / \mathrm{ml})$ for 24 h. $\alpha$-tubulin is used as an internal control (B)

Data are shown as the mean (SEM of three independent experiments). \#\#\# $p<0.001$ compared with the untreated group, ${ }^{*} p<0.05{ }^{* *} p<0.01$, and ${ }^{* * *} p<0.001$, compared with the vehicle group 
A
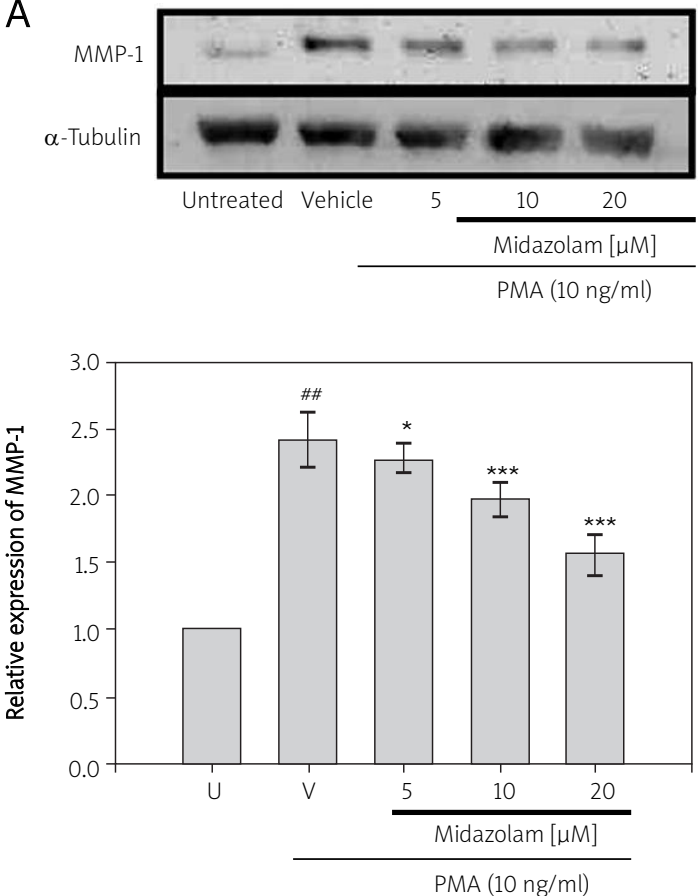

B
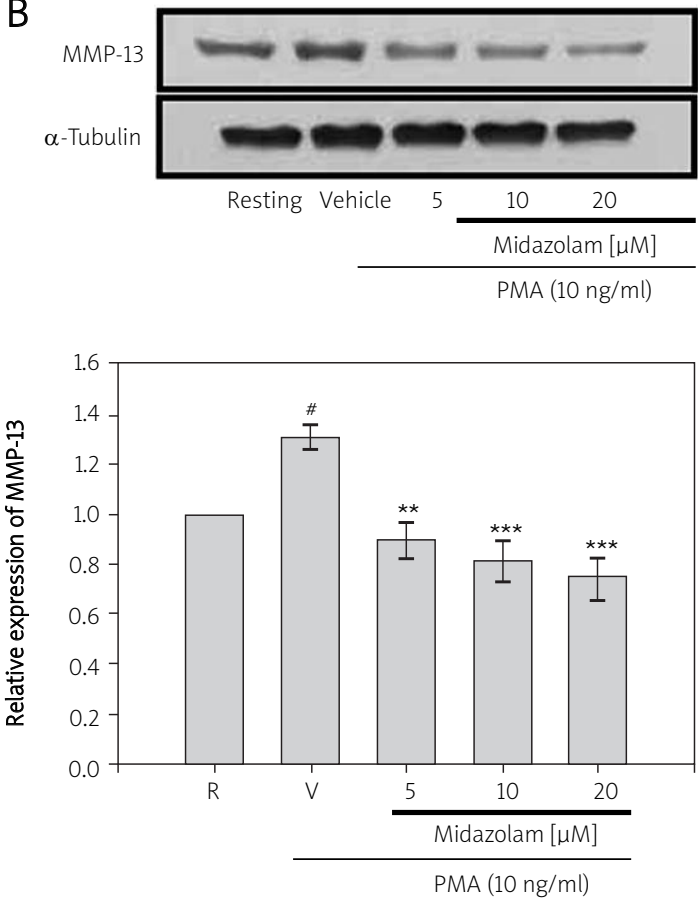

Figures 2. Effects of midazolam on phorbol-12myristate-13-acetate (PMA)-induced expression of MMP-1 (A) and MMP-13 (B) in chondrosarcoma SW1353 cell lines. Cell lysates were obtained and analyzed for MMP-1 and MMP-13 protein expressions by Western blotting. SW1353 cells $\left(2 \times 10^{6}\right.$ cells/ml in 6-well plates $)$ were treated with various concentrations of midazolam $(5 \mu \mathrm{M}, 10 \mu \mathrm{M}$, and $20 \mu \mathrm{M})$ or vehicle (DMSO) for $15 \mathrm{~min}$ before treatment with PMA $(10 \mathrm{ng} / \mathrm{ml})$ for $24 \mathrm{~h}$. $\alpha$-Tubulin is used as an internal control

Data are shown as the mean (SEM of three independent experiments). \#\#\#p<0.001 compared with the untreated group, ${ }^{*} p<0.05$, ${ }^{\star *} p<0.01$, and ${ }^{* \star *} p<0.001$, compared with the vehicle group
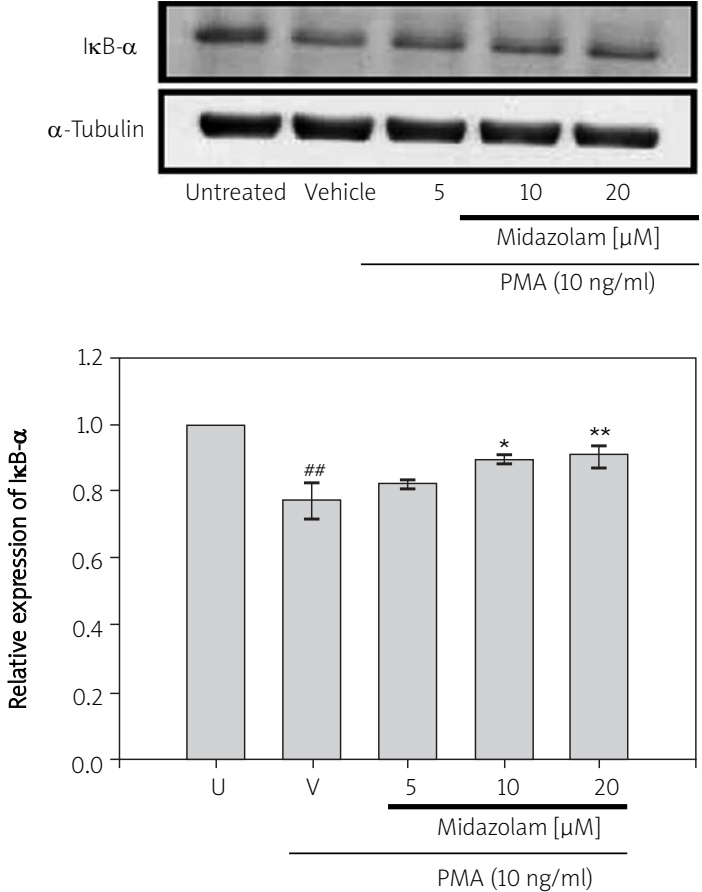

Figure 3. Effects of midazolam on phorbol-12myristate-13-acetate (PMA)-induced degradation of I $\mathrm{B}-\alpha$ in chondrosarcoma SW1353 cell lines. Cell lysates were obtained and analyzed for $1 \kappa B-\alpha$ protein degradation by Western blotting. SW1353 cells $\left(2 \times 10^{6}\right.$ cells $/ \mathrm{ml}$ in 6-well plates) were treated with various concentrations of midazolam $(5 \mu \mathrm{M}, 10 \mu \mathrm{M}$, and $20 \mu \mathrm{M}$ ) or vehicle (DMSO) for 15 min before treatment with PMA (10 ng/ml) for $24 \mathrm{~h}$. a-Tubulin is used as an internal control

Data are shown as the mean (SEM of three independent experiments). \#\#p<0.001 compared with the resting group, ${ }^{*} p<0.05$, and ${ }^{* *} p<0.01$, compared with the vehicle group

\section{Midazolam blocks PMA-mediated MAPK phosphorylation}

In order to examine whether midazolam affects stimulated ERK, p38, and JNK activation in SW-1353 cells, the phosphorylation levels of ERK, p38, and JNK were compared between the treatments with PMA in the presence or absence of midazolam. As shown in Figures 4 A, B, PMA (10 ng/ml) was found to significantly stimulate phos-ERK $(1.528 \pm 0.32$; $p<0.001)$ and phos-p38 (1.463 $\pm 0.41 ; p<0.01)$ levels, but treatment of cells with midazolam $(5 \mu \mathrm{M}$, $10 \mu \mathrm{M}$ and $20 \mu \mathrm{M})$ suppressed the PMA-induced levels of phos-ERK $(1.243 \pm 0.12,1.108 \pm 0.16$ and $0.903 \pm 0.19$, respectively) and phos-p38 (1.146 \pm 0.10 , $1.063 \pm 0.13$ and $0.946 \pm 0.18$, respectively). Moreover, the presence of midazolam at concentrations of $5 \mu \mathrm{M}$ and $10 \mu \mathrm{M}$ did not affect c-JNK levels and only a small impact was found at $20 \mu \mathrm{M}$ concentration (Data not shown). 


\section{Discussion}

The present study is the first to highlight the molecular evidence that midazolam inhibits MMPs in PMA-activated chondrocyte cells by blocking pERK, p38 MAPK and/or NF-KB-mediated transcriptional regulation. Cartilage chondrocytes have shown to be activated during the progression of arthritis in producing MMPs, resulting in the decomposition of local connective matrix [20]. Therefore, MMPs play an important pathological role as decomposition factors to induce cartilage damage during inflammation of OA, in particular, MMP-1, MMP-9 and MMP-13 [21]. Interestingly, phorbol ester is reported to induce some pathological stimulation through activating various signaling pathways in chondrocytes, and also could induce MMP-9, MMP-1 and MMP-13 as described in our earlier study [10]. The present study revealed that midazolam (5-20 $\mu M)$ markedly suppressed PMA-stimulated activation and expression of MMP-9 and expression of MMP-1 and MMP-13 in chondrocytes. In the range of approximately $3 \mu \mathrm{M}$ to $30 \mu \mathrm{M}$, midazolam suppressed expression of inducible nitric oxide synthase (iNOS) and cyclooxygenase-2 (COX-2), which are known as proinflammatory mediators. Plasma concentrations of benzodiazepines that have been used clinically ranged between $0.1 \mu \mathrm{M}$ and $50 \mu \mathrm{M}$ [22]. A study also found that in the range of 3-30 $\mu M$ midazolam suppresses VCAM-1 and monocyte adhesion in the TNF- $\alpha$ activated endothelial cell [23]. Midazolam at concentrations of 5-20 $\mu \mathrm{M}$ as used in the current study significantly inhibited activation and expression of MMP-9 and expression of MMP-1 and MMP-13. Amos et al. [24] demonstrated that the signaling of NF-KB and MAPKs could be activated under PKC stimulation.

The mechanism of down-regulating chondrocyte-specific matrix synthesis may also involve NF- $\kappa B$ activation [25]. It is apparent that OA synovial tissue has been considered as a typical marker

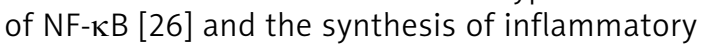
and destructive mediators from OA synovial tissue was reported to be NF- $\kappa B$ dependent [27]. The phosphorylation of $I_{\kappa} B$ and its subsequent degradation allows translocation of NF- $\kappa B$ to the nucleus. Agents that can down-modulate the activation of NF- $\kappa B$ have potential for therapeutic intervention. Kim et al. [28] discovered that midazolam exerted anti-inflammatory action by inhibiting iNOS and COX-2 expression, possibly through the suppression of NF- $\kappa$ B and p38 mitogen-activated protein kinase activation. In the present study, midazolam that had been administered to the PMA-stimulated chondrocytes could significantly inhibit $1 \kappa \mathrm{B}-\alpha$ degradation.

The MAPKs, including c-Jun N-terminal kinase, extracellular signal-regulated kinase, and p38, have been reported to play a critical role in cytokines and PMA-mediated MMP expression [10]. Both ERK and p38 MAPK activation are reported to be involved in
A
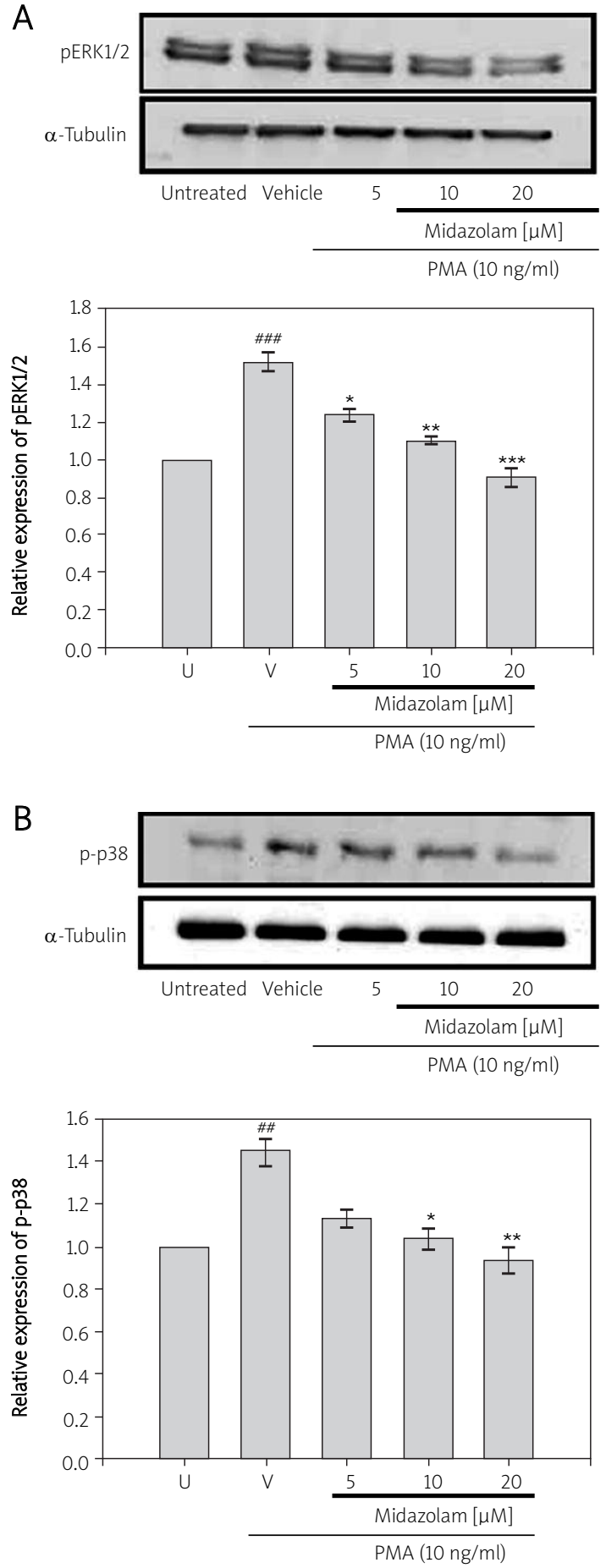

Figures 4. Effects of midazolam on phorbol-12myristate-13-acetate (PMA)-induced expression of pERK1/2 (A) and p38 (B) in chondrosarcoma SW1353 cell lines. Cell lysates were obtained and analyzed for pERK1/2 and p-p38 protein expression by Western blotting. SW1353 cells $\left(2 \times 10^{6}\right.$ cells/ml in 6-well plates) were treated with various concentrations of midazolam $(5 \mu \mathrm{M}, 10 \mu \mathrm{M}$, and $20 \mu \mathrm{M})$ or vehicle (DMSO) for 15 min before treatment with PMA $(10 \mathrm{ng} / \mathrm{ml})$ for 24 h. $\alpha$-Tubulin is used as an internal control

Data are shown as the mean (SEM) of three independent experiments. \#\#p<0.001 compared with the untreated group, ${ }^{*} p<0.05,{ }^{* *} p<0.01$ and ${ }^{* * *} p<0.001$, compared with the vehicle group 
stimulating MMP-9 production in chondrocytes [29]. In the present study, our results revealed that midazolam markedly attenuated PMA-induced phosphorylation of ERK and p38 MAPK, and these results are consistent with the cartilage breakdown study which revealed that cytokine increased chondrocyte MMP expression through a p38 MAPKmediated pathway [26]. It has previously been reported that midazolam inhibits $\mathrm{N}$-formyl-methionyl-leucyl-phenylalanine-induced p38 MAP kinase activation in neutrophils [30]. Consequently, inhibition of ERK and p38 MAPK by midazolam in this study may contribute to the reduction of MMP expression. Furthermore, midazolam also attenuated PMA-stimulated phosphorylation of ERK1/2, and this result was consistent with the study which found that the treatment of ERK inhibitor could reduce the production of PMAinduced MMPs in different human cells. Particularly, there was inhibitory action of midazolam on the ERK and p38 MAPK pathway, but no significant effect on the JNK MAPK pathway.

In OA, up-regulated MMPs are considered critical to degrading ECM. Therefore, MMPs would be reasonable therapeutic targets to treat osteoarthritis. In this study, midazolam markedly and dose-dependently decreased MMP-1, MMP-9, and MMP-13 levels by down-regulating MAP kinases, such as ERK-1/-2 and p38 kinase, and recovering $1 \kappa B-\alpha$ degradation, which could make midazolam more effective in preventing cartilage destruction. Ozok et al. [31] reported the efficacy of supplementation of midazolam to periprostatic nerve blockage (PNB) during probe insertion and needle penetration in patients for pain reduction. A clinical study has also shown that intra-articular midazolam injection could dramatically improve the degree of pain after knee arthroscopy [32]. Therefore, it is considerably relevant to this study as the rapid action of midazolam and its modest effects on chondrocytes help make it an effective and safe alternative for the treatment of arthritis patients.

In conclusion, the results of this study confirm that midazolam suppressed PMA-induced expression of MMP-9, MMP-1 and MMP-13 and these effects might be mediated via inhibition of $I_{\kappa} B-\alpha$ degradation and ERK/p38 MAPK activation. Since these responses are possibly cell specific, further experiments need to be explored to evaluate the potency of midazolam through in vivo animal and clinical models of arthritis. Depending on the outcome of this study, it may be appropriate to assess further the potential for midazolam to be developed as an anti-inflammatory and chondroprotective therapeutic for use in OA.

\section{Acknowledgments}

Jen-Jui Wang and Steven Kuan-Hua Huan contributed equally to this work. This work was sup- ported by grants from the National Science Council of Taiwan (NSC97-2320-B-038-016-MY3) and ChiMei Medical Center - Taipei Medical University (98CM-TMU-08).

\section{References}

1. LeGrand A, Fermor B, Fink C, et al. Interleukin-1, tumor necrosis factor alpha, and interleukin-17 synergistically up-regulate nitric oxide and prostaglandin E2 production in explants of human osteoarthritic knee menisci. Arthritis Rheum 2001; 44: 2078-83.

2. Kobayashi M, Tanzer M, Zukor DJ, Antoniou J, Feige U, Poole AR. Chondrocyte generated IL-1 and TNF are involved in matrix degradation of human osteoarthritis cartilage in explant culture. Arthritis Rheum 2002; 46: S80.

3. Tetlow LC, Adlam DJ, Woolley DE. Matrix metalloproteinase and proinflammatory cytokine production by chondrocytes of human osteoarthritic cartilage: associations with degenerative changes. Arthritis Rheum 2001; 44: 585-94.

4. Shi J, Schmitt-Talbot E, DiMattia DA, Dullea RG. The differential effects of IL-1 and TNF-alpha on proinflammatory cytokine and matrix metalloproteinase expression in human chondrosarcoma cells. Inflamm Res 2004; 53: 377-89.

5. Mengshol JA, Mix KS, Brinckerhoff CE. Matrix metalloproteinases as therapeutic targets in arthritic diseases: bull'seye or missing the mark? Arthritis Rheum 2002; 46: 13-20.

6. Malemud CJ. Matrix metalloproteinases: role in skeletal development and growth plate disorders. Front Biosci 2006; 11: 1702-15.

7. Mengshol JA, Vincenti MP, Coon Cl, Barchowsky A, Brinckerhoff CE. Interleukin-1 induction of collagenase 3 (matrix metalloproteinase 13) gene expression in chondrocytes requires p38, c-Jun $\mathrm{N}$-terminal kinase, and nuclear factor kappaB: differential regulation of collagenase 1 and collagenase 3. Arthritis Rheum 2000; 43: 801-11.

8. Shlopov BV, Lie WR, Mainardi CL, Cole AA, Chubinskaya S, Hasty KA. Osteoarthritic lesions: involvement of three different collagenases. Arthritis Rheum 1997; 40: 2065-74.

9. Knäuper V, Cowell S, Smith B, et al. The role of the C-terminal domain of human collagenase-3 (MMP-13) in the activation of procollagenase-3, substrate specificity, and tissue inhibitor of metalloproteinase interaction. J Biol Chem 1997; 272: 7608-16.

10. Lu YC, Jayakumar T, Duann YF, et al. Chondroprotective role of sesamol by inhibiting MMPs expression via retaining NF-kappaB signaling in activated SW1353 cells. J Agric Food Chem 2011; 59: 4969-78.

11. Shikhman AR, Kuhn K, Alaaeddine N, Lotz M. N-acetylglucosamine prevents IL-1beta-mediated activation of human chondrocytes. J Immunol 2001; 166: 5155-60.

12. Bondeson J, Brennan F, Foxwell B, Feldmann M. Effective adenoviral transfer of IkappaBalpha into human fibroblasts and chondrosarcoma cells reveals that the induction of matrix metalloproteinases and proinflammatory cytokines is nuclear factor-kappaB dependent. J Rheumatol 2000; 27: 2078-89.

13. Nishiyama T, Tamai H, Hanaoka K. Serum and cerebrospinal fluid concentrations of midazolam after epidural administration in dogs. Anesth Analg 2003; 96: 159-62.

14. Bazzichi L, Betti L, Giannaccini G, Rossi A, Lucacchini A. Peripheral-type benzodiazepine receptors in human mononuclear cells of patients affected by osteoarthritis, rheumatoid arthritis or psoriasic arthritis. Clin Biochem 2003; 36: 57-60.

15. Bribes E, Bourrie B, Esclangon M, Galiegue S, Vidal H, Casellas P. Involvement of the peripheral benzodiazepine 
receptor in the development of rheumatoid arthritis in Mrl/lpr mice. Eur J Pharmacol 2002; 452: 111-22.

16. Bartosikova L, Necas J, Bartosik T, Frana P, Pavlik M. Changes in biomechanical parameters during heart perfusion and after midazolam pre-medication - experimental pilot study. Biomed Pap Med Fac Univ Palacky Olomouc Czech Repub 2008; 152: 79-82.

17. Kelbel I, Weiss M. Anaesthetics and immune function. Curr Opin Anaesthesiol 2001; 14: 685-91.

18. Hsiao G, Huang HY, Fong TH, et al. Inhibitory mechanisms of YC-1 and PMC in the induction of iNOS expression by lipoteichoic acid in RAW 264.7 macrophages. Biochem Pharmacol 2004; 67: 1411-9.

19. Chung CL, Sheu JR, Liu HE, et al. Dynasore, a dynamin inhibitor, induces PAI-1 expression in MeT-5A human pleural mesothelial cells. Am J Respir Cell Mol Biol 2009; 40: 692-700.

20. Caterson B, Flannery CR, Hughes CE, Little CB. Mechanisms involved in cartilage proteoglycan catabolism. Matrix Biol 2000; 19: 333-44.

21. Yoshihara Y, Nakamura H, Obata K, et al. Matrix metalloproteinases and tissue inhibitors of metalloproteinases in synovial fluids from patients with rheumatoid arthritis or osteoarthritis. Ann Rheum Dis 2000; 59: 455-61.

22. Wilms H, Claasen J, Röhl C, Sievers J, Deuschl G, Lucius R. Involvement of benzodiazepine receptors in neuroinflammatory and neurodegenerative diseases: evidence from activated microglial cells in vitro. Neurobiol Dis 2003; 14: 417-24.

23. Joo HK, Oh SC, Cho EJ, et al. Midazolam inhibits tumor necrosis factor-alpha-induced endothelial activation: involvement of the peripheral benzodiazepine receptor. Anesthesiology 2009; 110: 106-12.

24. Amos N, Lauder S, Evans A, Feldmann M, Bondeson J. Adenoviral gene transfer into osteoarthritis synovial cells using the endogenous inhibitor IkappaBalpha reveals that most, but not all, inflammatory and destructive mediators are NFkappaB dependent. Rheumatology 2006; 45: 1201-9.

25. Murakami S, Lefebvre V, de Crombrugghe B. Potent inhibition of the master chondrogenic factor Sox 9 gene by interleukin-1 and tumor necrosis factor-alpha. J Biol Chem 2000; 275: 3687-92.

26. Marok R, Winyard PG, Coumbe A, et al. Activation of the transcription factor nuclear factor-kappaB in human inflamed synovial tissue. Arthritis Rheum 1996; 39: 583-91.

27. Baeuerle PA, Henkel T. Function and activation of NF-kappaB in the immune system. Annu Rev Immunol 1994; 12: $141-79$

28. Kim SN, Son SC, Lee SM, et al. Midazolam inhibits proinflammatory mediators in the lipopolysaccharide-activated macrophage. Anesthesiology 2006; 105: 105-10.

29. Grilli M, Chiu JJ, Lenardo MJ. NF-kappa B and Rel: participants in a multiform transcriptional regulatory system. Int Rev Cytol 1993; 143: 1-62.

30. Ghori K, O'Driscoll J, Shorten G. The effect of midazolam on neutrophil mitogen-activated protein kinase. Eur J Anaesthesiol 2010; 27: 562-5.

31. Ozok HU, Sagnak L, Ates MA, Karakoyunlu N, Topaloglu H, Ersoy $\mathrm{H}$. The efficiency of a sedative or analgesic supplement to periprostatic nerve blockage for pain control during transrectal ultrasound-guided prostate biopsy a prospective, randomized, controlled, double blind study. Arch Med Sci 2010; 6: 787-92.

32. Batra YK, Mahajan R, Kumar S, Rajeev S, Singh Dhillon M. A dose-ranging study of intraarticular midazolam for pain relief after knee arthroscopy. Anesth Analg 2008; 107: $669-72$. 